\title{
Thermo-optic Tunable Optical Filter Based on Fabry-Perot Microcavities in SOI
}

\author{
Zhe $\mathrm{Li}^{\mathrm{a}}$, Huajuan $\mathrm{Qi}^{\mathrm{b}}$, Yongchuan Xiao ${ }^{\mathrm{c}}$ and Fengli Gao ${ }^{\mathrm{d}}$ \\ State Key Laboratory on Integrated Optoelectronics, College of Electronic Science and Engineering, \\ Jilin University, Changchun, 130012, China \\ aileezhe@gmail.com, bhuajuan1988@yahoo.con, xiaoyc10@mails.jlu.edu.cn \\ dCorresponding author, gaofl@jlu.edu.cn
} Keywords: Tunable Optical Filter ; Thermo-optic Effect; Waveguide; Transfer Matrix Method;
Fabry-Perot Microcavities.

\begin{abstract}
An integrated TOF (Tunable Optical Filter) based on thermo-optic effect in Silicon on insulator (SOI) rib waveguide is designed and simulated. The device is comprised of two high refractivity contrast $\mathrm{Si}$ /Air stacks, functioning as high reflectivity of DBRs and separated by a variable refractive index Si F-P cavity. The output characteristics are calculated and simulated based on Transfer Matrix Method (TMM). Wavelength tuning is achieved through thermal modulation of refractive variation of the cavity.As the cavity $\mathrm{Si}$ is heated,the refractive index of the cavity increases. When the temperature of cavity $\mathrm{Si}$ changes within $100^{\circ} \mathrm{C}$, the central wavelength gets a continuous $8 \mathrm{~nm}$ shift from $1550 \mathrm{~nm}$ to $1558 \mathrm{~nm}$, which is right located in the WDM (Wavelength division multiplexing) networks operating at $\mathrm{C}$-band. Moreover, by calculating, the tuning sensitivity is about $0.08 \mathrm{~nm} /{ }^{\circ} \mathrm{C}$. Owing to the compact size and excellent characteristics of integration, the proposed component has a promising utilization in spectroscopy and optical communication.
\end{abstract}

\section{Introduction}

It is acknowledged that tunable optical filter (TOF) is quite an important component in WDM system which is the dominant technology in large-capacity optical networks [1]. TOF whose frequency characteristics can be tailored to a desired response is an enabling technology for exploiting the full bandwidth potential of optical fiber communication systems [2]. As a consequence, it has attracted lots of attention in both existed telecommunication network and all-optical network. Conventional TOFs usually fall into several following categories: acousto-optic TOF [3], electro-optic TOF [4], thermo-optic TOF [1], dielectric thin-film interference TOF [5], fiber Bragg grating TOF [6], Mach-Zehnder interferometer TOF [7] and Fabry-Perot interferometer TOF [8]. An ideal filter can provide excellent selectivity of the central wavelength, wide range of tuning, low insert loss and chromatic dispersion so that it can be well applied to the WDM system [9]. On account of the outstanding optical wavelength resolution, Fabry-Perot filters are considered as essential instruments and promising candidates for WDM system.

In this paper, we investigate a tunable optical filter based on Fabry-Perot resonator on SOI rib waveguide with $2.5 \mathrm{Si} /$ Air pairs and $4.93 \mu \mathrm{m}$ cavity length. A filter with $66.2 \mathrm{~nm}$ FSR and $0.05 \mathrm{~nm}$ FWHM is proposed and the finesse is exceeding 1000. Benefited from the thermo-optical coefficient of $\mathrm{Si}$, the filter can realize continuous wavelength tuning ranging from $1550 \mathrm{~nm}$ to $1558 \mathrm{~nm}$ within $100^{\circ} \mathrm{C}$ temperature variation of cavity $\mathrm{Si}$. The good performance guarantees a promising application in both spectroscopy and optical communication. In addition, compared with exotic materials such as the III-V compounds or the insulator lithium niobate $\left(\mathrm{LiNbO}_{3}\right), \mathrm{Si}$ is a kind of well-understood and robust material, compatible with the manufacturing in the microelectronics industry and potential for straightforward integration with electrical components [10]. 


\section{Principle and design}

Since the device is designed and manufactured on SOI wafers, before we introduce the specific structure and principles of the filter, there is still an important aspect remained to be taken into consideration - single mode transmission condition of the SOI rib waveguide. Figure 1(a) shows the cross section of the schematic of the waveguide [11].

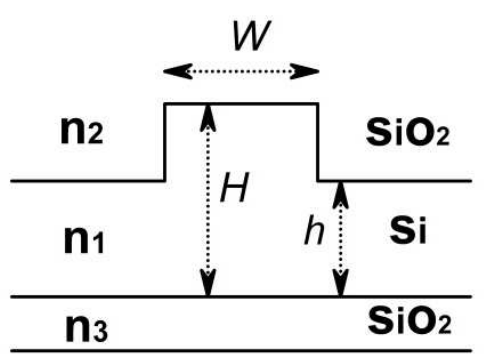

Figure 1(a). Waveguide cross section

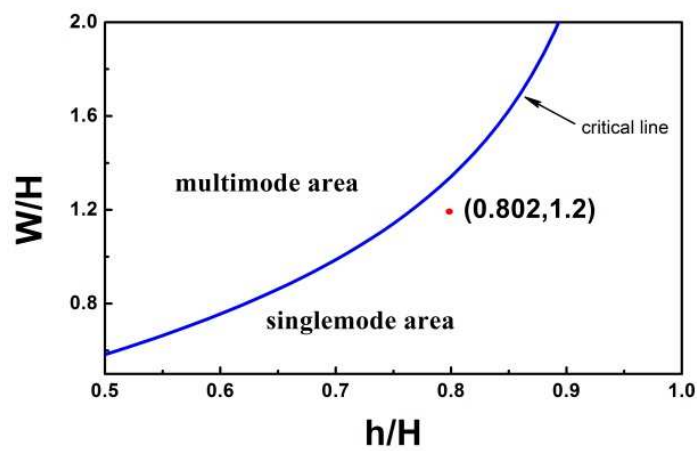

Figure 1(b). Single-mode critical condition of SOI rib waveguide

From Figure 1(a), for $H=5 \mu \mathrm{m}$ top Si, external rib height of $4.1 \mu \mathrm{m}$ and waveguide rib width of $6 \mu \mathrm{m}$ are considered to ensure single-mode transmission. The critical line of single mode propagation condition and the selected point of detailed waveguide dimension obtained by EIM (Effective index method) are plotted in Figure 1(b). The simulated results of the optical field of this single mode waveguide are shown in Figure 2. Here, Figure 2(b) is its 3D profile. Obviously, the only existed fundamental mode field is confined in core.

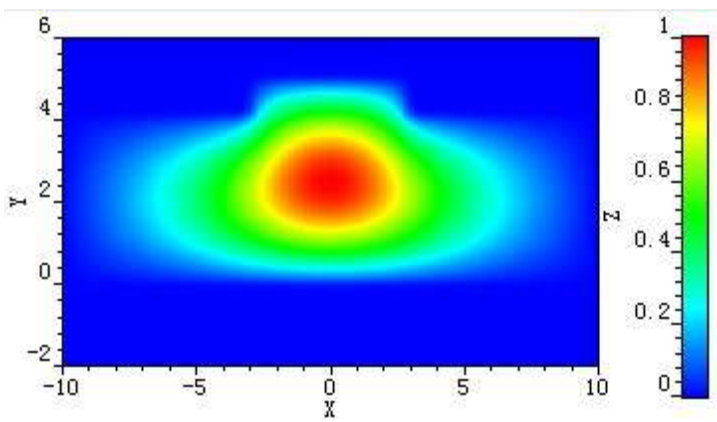

Figure 2(a). Single mode field of the rib waveguide

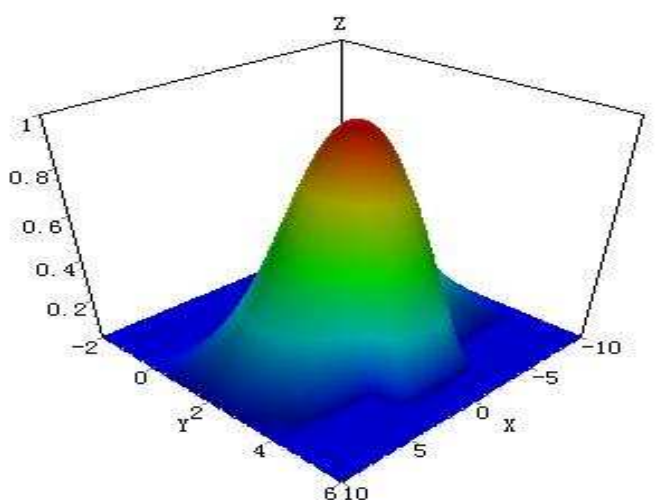

Figure 2(b). 3D image of the single mode

It is reported that there are various methods to realize a waveguide F-P cavity narrowband filter, such as waveguide grating and photonic crystal technologies. Most of them can adopt Airy functions to describe the input and output transmission characteristics of the F-P cavity, which is expressed as follows:

$$
T_{\text {Airy }}=\frac{1}{1+\left[\frac{2 F}{\pi} \sin \left(\delta_{c}\right)\right]^{2}}
$$

Where, $F=\pi \sqrt{R} /(1-R)$ is reflectivity finesse; $R$ is the reflectivity on both sides of the cavity; $\delta_{c}=2 \pi n_{e}(\lambda) L_{e} / \lambda$ is phase factor which is related to the effective refractive index; $L_{c}$ is the length of the cavity and $\lambda$ is the operation wavelength. From formula (1), an appropriate length of the cavity and high reflectivity on both sides of the cavity should be provided so that $F$ factor could be improved to construct a narrowband filter. 
The schematic of the thermo-optical tunable filter is shown in Figure 3, which includes a F-P resonant cavity and two DBRs consisting of $2.5 \mathrm{Si} /$ Air pairs. The thickness of the each layer of DBRs and the length of F-P cavity are acquired by the following equations [1]:

$$
\begin{gathered}
W_{s i}=\left(\frac{1}{2} N_{s}+\frac{1}{4}\right) \frac{\lambda}{n_{s}} \\
W_{\text {air }}=\left(\frac{1}{2} N_{a}+\frac{1}{4}\right) \frac{\lambda}{n_{a}} \\
L_{c}=\left(\frac{N_{c}}{2}\right) \frac{\lambda}{n_{c}}
\end{gathered}
$$

Where, $W_{\text {si }}$ and $W_{\text {air }}$ are the width of the designed Si and air of the DBRs, respectively; $L_{c}$ is the length of the proposed Si resonant cavity. $N_{s}, N_{a}$ and $N_{c}$ are the integer which are related to actual fabrication; $\lambda$ is the central wavelength; $n_{s}, n_{a}$ and $n_{c}$ are the effective refraction index of the Si core, air of DBRs and Si cavity, respectively.

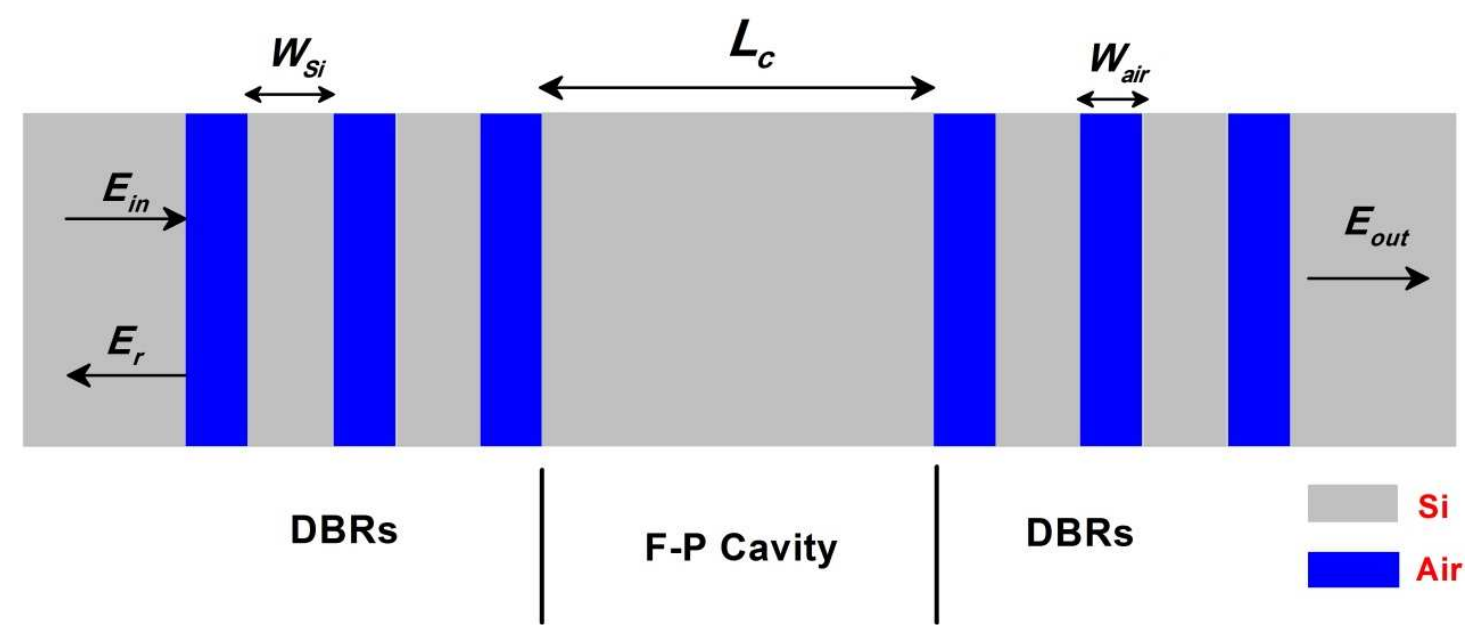

Figure 3. Plane view of the Fabry-Perot interferometer TOF

In order to get suitable structure and dimension, the DBRs and F-P cavity are investigated using transfer matrix method. It is expressed as below [12]:

$$
\begin{gathered}
{\left[\begin{array}{l}
B \\
C
\end{array}\right]=\prod_{m=1}^{k}\left[\begin{array}{cc}
\cos \delta_{m} & \mathrm{i} \cdot \sin \delta_{m} / n_{\text {em }}(\lambda) \\
\mathrm{i} \cdot n_{\text {em }}(\lambda) \cdot \sin \delta_{m} & \cos \delta_{m}
\end{array}\right] \cdot\left[\begin{array}{c}
1 \\
n_{\text {esub }}(\lambda)
\end{array}\right]} \\
Y=\frac{C}{B} \quad r^{*}=\left(\frac{n_{\text {einc }}(\lambda)-Y}{n_{\text {einc }}(\lambda)+Y}\right)\left(\frac{n_{\text {einc }}(\lambda)-Y}{n_{\text {einc }}(\lambda)+Y}\right)^{*} \\
r=\frac{E_{r}}{E_{\text {in }}}=\frac{n_{\text {einc }}(\lambda)-Y}{n_{\text {einc }}(\lambda)+Y}, R=r n_{\text {einc }}(\lambda)\left(Y+Y^{*}\right) \\
t=\frac{E_{\text {out }}}{E_{\text {in }}}=\frac{2 n_{\text {einc }}(\lambda)}{n_{\text {einc }}(\lambda)+Y}, T=1-R=\frac{\left(n_{\text {einc }}(\lambda)+Y\right)^{*}}{\left(n_{\text {einc }}(\lambda)+Y\right)\left(n_{\text {en }}\right.}
\end{gathered}
$$

where $\lambda$ is the operation wavelength; $d_{m}$ is the thickness of m-th layer; $n_{\text {einc }}(\lambda), n_{\text {em }}(\lambda)$ and $n_{\text {esub }}(\lambda)$ represent the effective refractive index of the incident medium, the $m$-th layer and output medium at different wavelengths; $R$ is the reflectance of the DBRs and $T$ is the transmittance of the device. Equations $(5) \sim(8)$ have taken the material dispersion and the waveguide dispersion of the SOI into account, thus the calculation is more reliable, which can achieve the reliable reference data for the actual production.

Considering the tuning in C-band and low insert loss, $4.93 \mu \mathrm{m}$ cavity length is chosen, where the result of $66.2 \mathrm{~nm}$ FSR is shown in Figure 4(a). The final transmission characteristic is also 
demonstrated in Figure 4 (a). Based on expression (7), large reflectivity must be guaranteed for perfect wavelength selectivity, and so DBRs play a key role. A comparison of reflectivity between 2.5 and 3.5 Si/Air pairs of DBR has been conducted, whose result is shown in Figure 4 (b).

Hence, considering the actual limitation of integrated silicon processing, transmission loss and the character of F-P resonant, we choose $N_{s}=2, N_{a}=0$ and $N_{c}=11$ as the optimized value. That is to say, $W_{s i}=560 \mathrm{~nm}, W_{\text {air }}=380 \mathrm{~nm}$ and $L_{c}=4.93 \mu \mathrm{m}$. Owing to the appropriate dimension, the finesse of mentioned device is up to 1364.

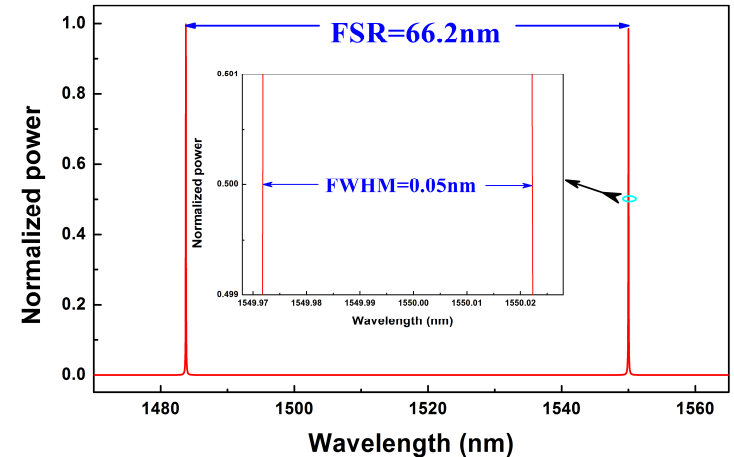

Figure 4(a). Static filter characteristics and FWHM demonstration with 2.5 pairs of Si/Air stacks.

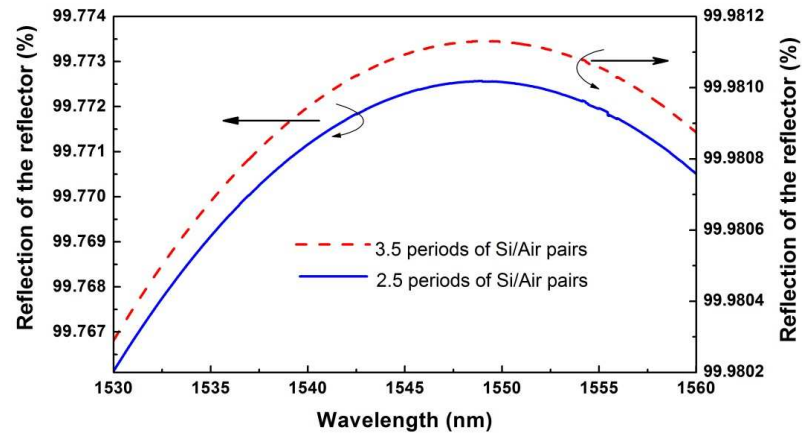

Figure 4(b). The reflectivity of DBRs varies from different pairs of $\mathrm{Si} /$ Air.

Simultaneously, the transmission spectrums in the condition of refractive index changing caused by heating the cavity $\mathrm{Si}$ is simulated numerically. As that is shown in Figure 5(a), it indicates that when the cavity $\mathrm{Si}$ is heated from $25^{\circ} \mathrm{C}$ to $125^{\circ} \mathrm{C}$ with approximation 0.0184 refraction index variation, the central wavelength gets a continuous $8 \mathrm{~nm}$ shift from $1550 \mathrm{~nm}$ to $1558 \mathrm{~nm}$ where the optical telecommunication windows are located. Also, from Figure 5(b), the tuning sensitivity is approximately $0.08 \mathrm{~nm} /{ }^{\circ} \mathrm{C}$ on the average. These periodic arrangements of different dielectric materials offer a huge potential to make wavelength selective optical devices be applied to the current WDM optical telecommunication networks.

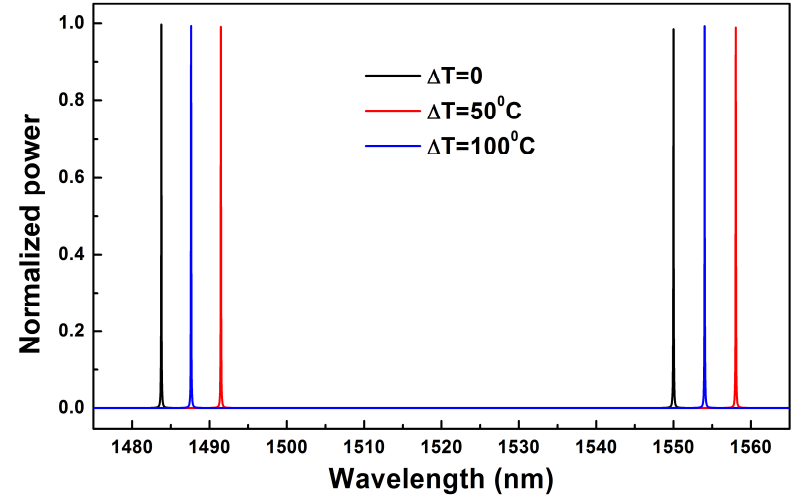

Figure 5(a). The transmission spectrum of thermal modulation

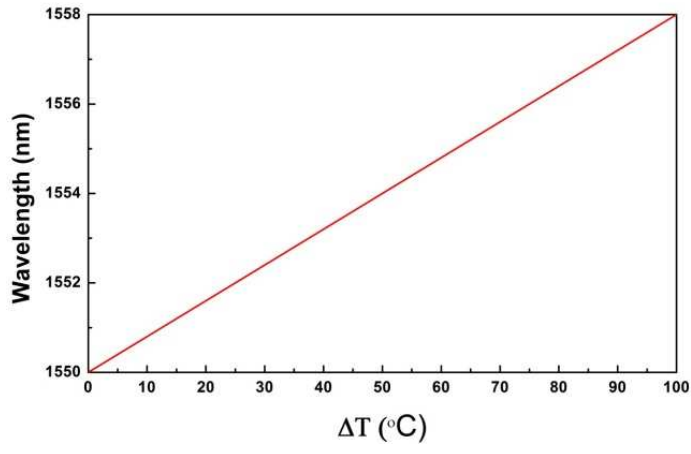

Figure 5(b). The temperature related wavelength adjustment of filter

Finally, in order to provide $100^{\circ} \mathrm{C}$ temperature variation, we provide thermal analysis by using ANSYS. When $2130 \mu \mathrm{W} / \mu \mathrm{m}^{3}$ thermal power flow is applied on $200 \mathrm{~nm}$ thick Pt electrodes, the steady-state temperature distribution is shown in Figure 6(a). Also, the temperature diversification in $\mathrm{X}$-direction and $\mathrm{Y}$-direction are shown in Figure 6(b) and Figure 6(c), respectively, where we can see that $100^{\circ} \mathrm{C}$ temperature variation in the center of SOI waveguide is clear. The following Figure 7 shows the transient characteristics of the proposed device, which features approximate $100 \mu$ s of response time, and it indicates that a high-speed response can be guaranteed if it is applied to WDM. 


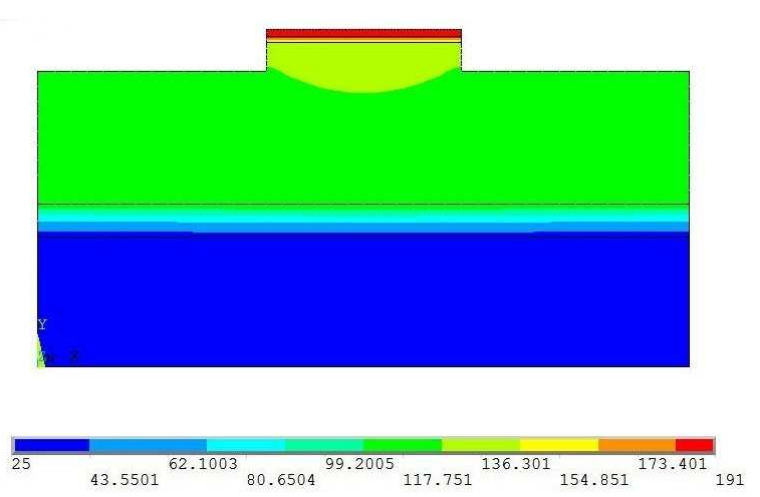

Figure 6(a). Steady-state temperature distribution

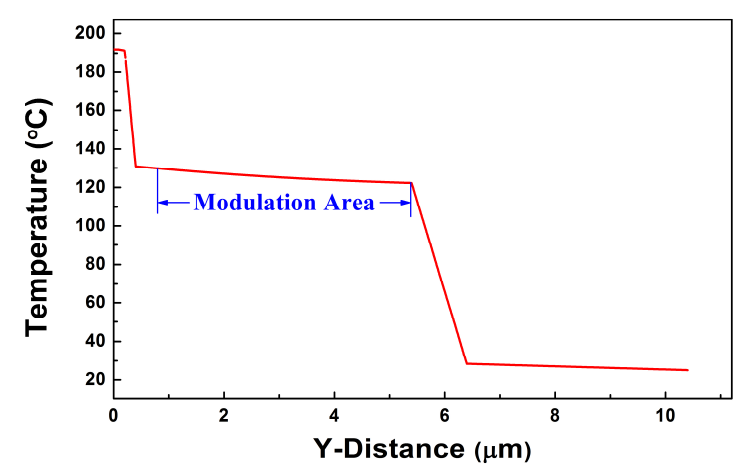

Figure 6(c). Y-direction temperature distribution

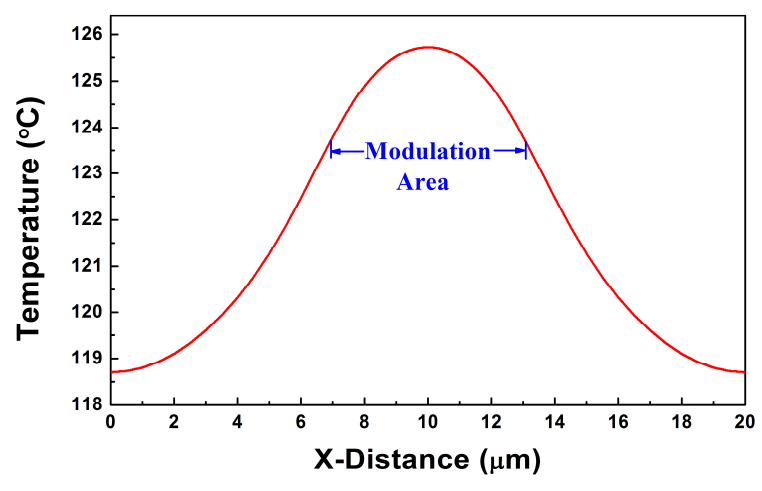

Figure 6(b). X-direction temperature distribution

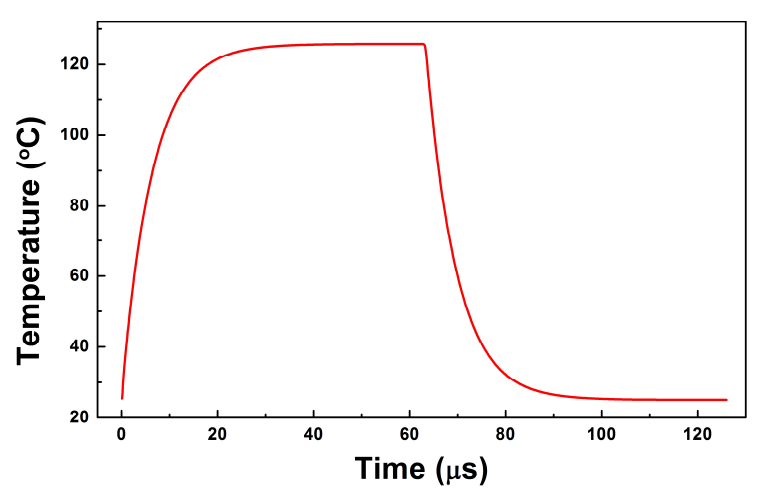

Figure 7. Response time of the device

\section{Summary}

We propose a $0.05 \mathrm{~nm}$ FWHM, $8 \mathrm{~nm}$ tuning range and $0.08 \mathrm{~nm} /{ }^{\circ} \mathrm{C}$ sensitivity F-P filter based on both sides of Si/Air DBRs, which is separated by a variable refractive index of Si F-P cavity on SOI rib waveguides for telecommunication applications. By simulation, we get $0.05 \mathrm{~nm}$ FWHM at $1550 \mathrm{~nm}$ wavelength and $66.2 \mathrm{~nm}$ FSR by using 2.5 pairs of Si/Air multilayers on each side of the resonator with $4.93 \mu \mathrm{m}$ cavity length. In theory, by heating, $8 \mathrm{~nm}$ wavelength tuning ranging from $1550 \mathrm{~nm}$ to $1558 \mathrm{~nm}$ and $100 \mu$ s response time can be realized because of the large thermo-optic coefficient of Si. Moreover, it is very easy to integrate the Si-based component with other functional devices.

\section{Acknowledgements}

The authors are grateful to Science and Technology Development Plan of Jilin Province (Grant No.20110314), the National Natural Science Foundation of China (Grant No.61077046) and the Basic Scientific research Fund of Jilin University (Grant No.200903084) for the support in the work.

\section{References}

[1] Sung-Sik Yun and Jong-Hyun Lee 2003 J. Micromech. Microeng. 13721

[2] Stone J and Stulz L W 1987 Electron. Lett. 23 781-3

[3] H. S. Kim, S. H. Yun, I. K. Kwang, and B. Y. Kim, 1997 Opt. Lett. 22, 1476-1478

[4] A d'Alessandro, D Donisi, L De Sio, R Beccherelli, R Asquini, R Caputo, and C Umeton 2008 Opt. Express 16 9254-9260

[5] Stéphane Larouche and Ludvik Martinu 2008 J. Appl. Phys. 47 C219-C230 
[6] Allan C L, Wong, Marco Giovinazzo, Hwa-Yaw Tam, Chao Lu, and Gang-Ding Peng $2010 \mathrm{~J}$. Lightwave Technol. 28 898-904

[7] Stone J and Stulz L W 1987 Electron. Lett. 23 781-3

[8] Wooten L, Stone R L, Miles W and Bradly E M 1996 J. Lightwave Technol. 14 2530-6

[9] Zhengxuan Li, Lilin Yi, Yan Zhang, Yi Dong, Shilin Xiao and Weisheng Hu 2012 Photonics Technology Letters, IEEE PP 1-1

[10] Mutsunori Uenuma and Teruaki Motooka 2009 Opt. Lett. 34 599-601

[11]Pogossian, S P, L Vescan and A Vonsovici 1998 Lightwave Technology Journal 16 1851-1853

[12] Weidong S, L Xiangdong, H Biqin, Z Yong, L Xu and G Peifu 2004 Optics Communications 239 153-160 